\title{
The Design of Motor Control System in Portable Ventilators
}

\author{
Bin Chen \\ Faculty of Information Science and Engineering \\ Ningbo University \\ Ningbo, China \\ nbuchenbin@163.com
}

\begin{abstract}
This paper introduces a design of brushless motor control system in portable ventilators, which is based on ML4425 and C8051F330 microcontroller. For the simplifycation in circuit design and programming, this is very suitable for portable ventilators. In the design, the ML4425 motor controller provides all of the functions necessary for starting and controlling the speed of delta or triangle wound brushless DC (BLDC) motors without sensors. And the microcontroller is responsible for much less tasks to improve real-time performance. With the detailed explanation of step-by-step implementation, the design is able to provide significant efficiency improvements, lower acoustic noise and better reliability.
\end{abstract}

\section{Keywords- BLDC motor; contol system; ML4425; PWM}

\section{INTRODUCTION}

In present, BLDC motors are replacing brush motors in numerous applications as they offer significant energy efficiency improvements, lower acoustic noise and better reliability to name a few advantages. Especially in the field of portable ventilators, BLDC motors are much more widely used than ever [1]. Portable ventilators are compact devices designed to provide mechanical ventilation where piped gases and mains electricity are unavailable [2].

Generally, a BLDC motor drive uses one or more sensors giving positional information to keep synchronization. For cost and technical reasons, the BLDC sensorless drive is an essential capability of a brushless motor controller. Based on different system architectures, there're some kinds of BLDC control systems. For instance, ARM micro-controllers [3] and DSP chips [4] are widely used in the system to achieve good performance. For the complexity in circuit design and programming, they're not suitable for the control system in portable ventilators.

ML4425, a kind of motor controllers, provides all the circuitry for sensorless speed control of 3-phase BLDC motors [5]. The functions include start-up circuitry, back electromotive force (BEMF) commutation control, PWM speed control, current limiting, and undervoltage protection. For this reason, it allows various implementations of sensorless BLDC control with the lowest possible system cost while maintaining the highest performance [6]. This paper describes the design of a better BLDC control system, which is based on ML4425 and C8051F330 microcontroller. In this system, the microcontroller is responsible for much less tasks to improve the real-time performance.
Hong Li

Faculty of Information Science and Engineering Ningbo University

Ningbo, China

lihong2@nbu.edu.cn

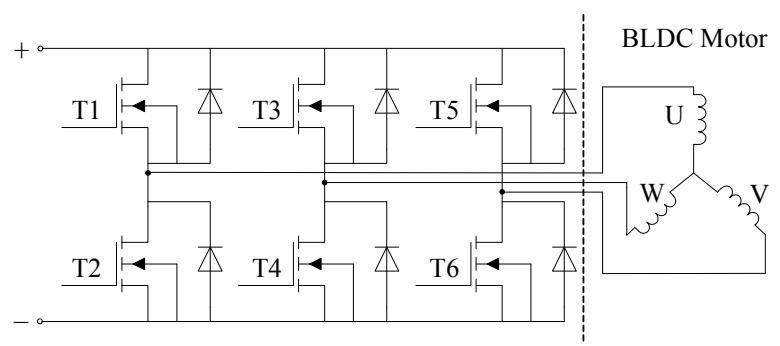

Figure 1. The BLDC motor drive schematic.

\section{PRINCIPLE OF MOTOR CONTROL}

To be driven and controlled properly, 3-phase brushless motors require a 3 half bridge "inverter" topology to deliver a 6-step or sine wave signal [7]. As shown in Fig. 1. They also require the electronic commutation of motor phases to respect the synchronization between statoric flux and the permanent magnet of the rotor.

There are many categories of sensorless control strategies. However, the most popular category is based on the detection of the BEMF induced by the movement of a permanent magnet rotor in front of stator winding. The category uses the zero crossing of BEMF to synchronize phase commutations.

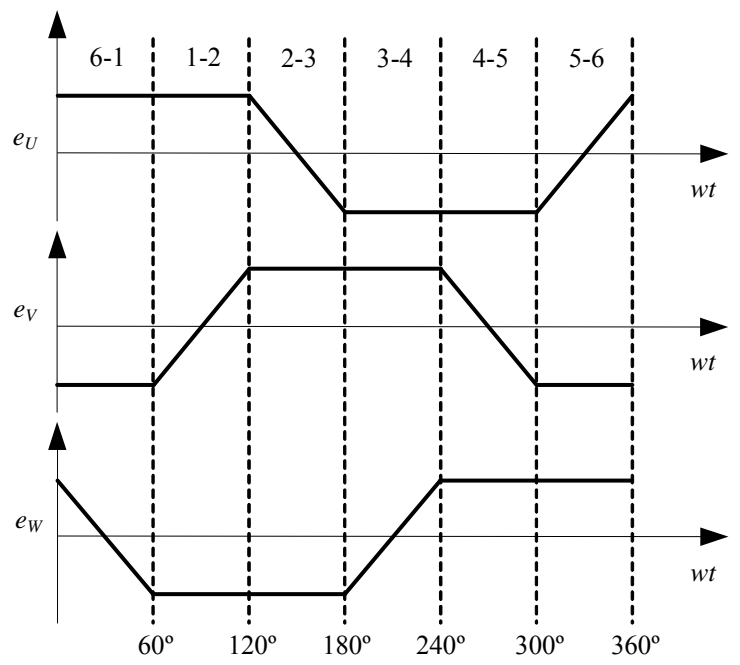

Figure 2. BEMF waveforms. 
This method requires the use of a trapezoidal signal in order to have a zero crossing of the BEMF [8]. The typical trapezoidal BEMF waveforms and corresponding driving voltages of a 3-phase BLDC are shown in Fig. 2. In every commutation step, one phase winding is connected to positive supply voltage, one phase winding is connected to negative supply voltage and one phase is floating. The BEMF in the floating phase will result in a zero crossing when it crosses the average of the positive and negative supply voltage.

The zero crossing occurs right in the middle of two commutations. At constant speed, or slowly varying speed, the time period from one commutation to zero-crossing and the time period from zero-crossing to the next commutation are equal. This is used as basis for this implementation of sensorless commutation control.

\section{DeSign OF THE CONTROL SyStem}

In the design of system, the guiding principle is simplification in hardware and software. Therefore, this design is based on ML4425 and C8051F330 microcontroller. Almost all tasks about motor controlling are assigned to ML4425. Besides, C8051F330 microcontroller acts as the main controller in design to manage the whole system. The block diagram of system hardware is shown in Fig. 3.

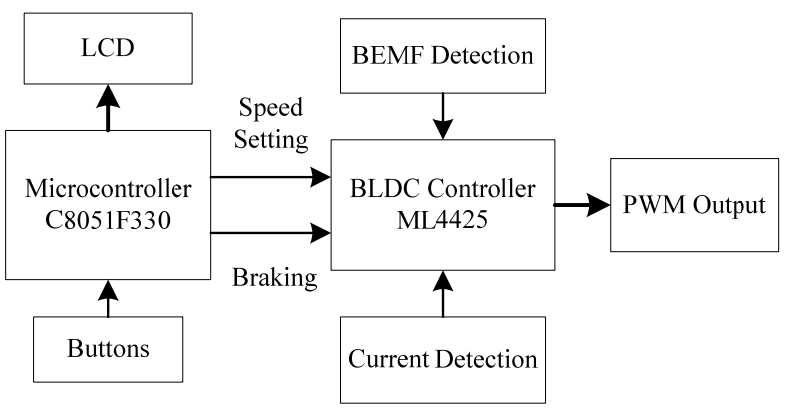

Figure 3. The block diagram of system hardware .

Due to the use of the motor controller chip, the system is much simpler than others. On one hand, C8051F330 is mainly responsible for human-machine interaction. Through the buttons, para-meter values, e.g. speed of motor, could be set by users. Besides, there're two signals connecting between C8051F330 and ML4425. One is speed setting that can create a PWM duty cycle to control the speed. The other one is braking signal, which is able to turn drivers off. On the other hand, ML4425 provides all of the functions necessary for starting and controlling the speed of delta or triangle wound BLDC motors without sensors. And BEMF voltage is sensed from the motor windings to determine the proper commutation phase sequence using a phase locked loop (PLL). This will commutate a wide range of 3-phase BLDC motors and is insensitive to PWM noise and motor snubbing circuitry.

\section{A. Design of the Motor Control Circuit}

The ML4425 provide sensorless commutation plus on chip PWM speed control for brushless DC motors. An integ-

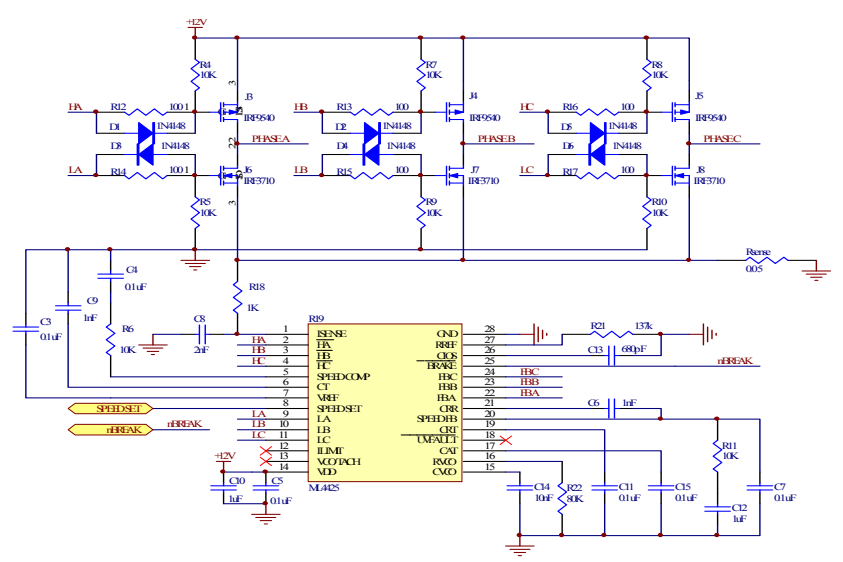

Figure 4. The control circuit based on ML4425 .

rating BEMF sensor, combined with an on-chip voltage controlled oscillator (VCO) and sequencer, form a PLL. And the parts provide on chip braking and power failure detection. The main control circuit is shown in Fig. 4.

In the design, the part number of BLDC motor is WS7040 as shown in TABLE I.

TABLE I. SPECIFICATION OF BLDC MOTOR

\begin{tabular}{|l|c|c|}
\hline \multicolumn{1}{|c|}{ Item } & Specification & Unit \\
\hline Part No & WS7040-12 & $/$ \\
\hline Operating Voltage Range & $10-13.8$ & $\mathrm{~V}$ \\
\hline Speed & 32000 & RPM \\
\hline Current & 3.5 & $\mathrm{~A}$ \\
\hline
\end{tabular}

According to the datasheet, ML4425 can drive external $\mathrm{P}$ and $\mathrm{N}$ channel MOSFET directly in $12 \mathrm{~V}$ applications. For that, IRF3710 (N channel) and IRF9540 (P channel) are used in the design to drive the motor shown in Fig. 4. They have advantages of extremely low on-resistance and high continuous source current to provide high efficiency and great reliability.

1) Start-up Implementation

When power is applied to the ML4425 and the motor is at rest, the BEMF is equal to zero. The motor needs to be rotating for the BEMF sampler to lock onto the rotor position and commutate the motor. The ML4425 uses an open loop start-up technique to bring the rotor from rest up to a speed fast enough to allow BEMF sensing.

Start-up is comprised of three modes: align mode, ramp mode, and run mode.

\section{a) Align Mode}

Before the motor can be started, the rotor must be in a known position. When power is first applied to the ML4425, the controller is reset into the align mode. Align mode turns on the output drivers LB, HA, and $\mathrm{HC}$ which aligns the motor into a position 30 electrical degrees before the center of the first commutation state. This is shown as state $\mathrm{R}$ in TABLE II. 
TABLE II. COMMUTATION STATE FUNCTIONS

\begin{tabular}{|c|c|c|c|c|c|c|c|}
\hline \multirow{2}{*}{ State } & \multicolumn{6}{|c|}{ Output } & \multirow{2}{*}{$\begin{array}{c}\text { Input } \\
\text { Sampling }\end{array}$} \\
\hline & $L A$ & $L B$ & $L A$ & $H A$ & $H B$ & HC & \\
\hline $\mathrm{R}$ & OFF & ON & OFF & ON & OFF & ON & 1 \\
\hline A & OFF & OFF & ON & ON & OFF & OFF & FB B \\
\hline B & OFF & OFF & ON & OFF & ON & OFF & FB A \\
\hline $\mathrm{C}$ & $\mathrm{ON}$ & OFF & OFF & OFF & $\mathrm{ON}$ & OFF & FB C \\
\hline D & $\mathrm{ON}$ & OFF & OFF & OFF & OFF & ON & FB B \\
\hline E & OFF & ON & OFF & OFF & OFF & ON & FB A \\
\hline F & OFF & ON & OFF & ON & OFF & OFF & FB C \\
\hline
\end{tabular}

Align mode must last long enough to allow the motor and its load to settle into this position. The align mode time is set by a capacitor connected to the $\mathrm{C}_{\mathrm{AT}}$ pin. $\mathrm{C}_{\mathrm{AT}}$ is charged by a constant $750 \mu \mathrm{A}$ current from GND to $1.5 \mathrm{~V}$ until the align comparator trips to end the align mode. A starting point for $\mathrm{C}_{\mathrm{AT}}$ is calculated as follows:

$$
C_{A T}=\frac{t_{S} \times 7.5 \times 10^{-7} \times a m p}{1.5 \mathrm{~V}}
$$

If the align time is not long enough to allow the rotor to settle for reliable starting, then increase $\mathrm{C}_{\mathrm{AT}}$ until the desired performance is achieved.

\section{b) Ramp Mode}

At the end of align mode the controller goes into ramp mode. Ramp mode starts commutating through the states A through $\mathrm{F}$ as shown in TABLE II. It ramps up the commutation frequency for a fixed length of time. This allows the motor to reach a sufficient speed for the BEMF sampler to lock commutation onto the motor's BEMF. The amount of time the ML4425 stays in ramp mode is determined by a capacitor connected to the $C_{R T}$ pin. $C_{R T}$ is charged by a constant $750 \mu \mathrm{A}$ current from GND to $1.5 \mathrm{~V}$ until the ramp comparator trips to end the ramp mode. This gives a fixed ramp time. $\mathrm{C}_{\mathrm{RT}}$ is calculated as follows:

$$
C_{R T}=\frac{2 \pi \times J \times 5 \times 10^{-7} \times a m p \times K_{\mathrm{V}}}{I_{M A X} \times K_{t} \times 3 \times N}
$$

The rate at which the ML4425 ramps up the motor speed is determined by a fixed $500 \mu \mathrm{A}$ current source on the SPEED FB pin. The current sources charges up the PLL filter components causing the VCO frequency to ramp up. During ramp mode, the BEMF sampler is disabled to allow control of the ramping to be set only by the $500 \mu \mathrm{A}$ current source.

\section{c) Run Mode}

At the end of ramp mode the controller goes into run mode. In run mode, the BEMF sensing is enabled and commutation is now under the control of the phase locked loop. Motor speed is now regulated by the speed control loop.

\section{2) Speed Control}

Speed control is accomplished by setting a speed command at SPEED SET pin with an input voltage from 0 to $6.9 \mathrm{~V}\left(\mathrm{~V}_{\mathrm{REF}}\right)$. The accuracy of the speed command is determined by the external components $\mathrm{R}_{\mathrm{VCO}}$ and $\mathrm{C}_{\mathrm{VCO}}$.
There are a number of methods that can be used to control the speed command of the ML4425. One is to use a $10 \mathrm{k} \Omega$ potentiometer from $V_{\text {REF }}$ to ground with the wiper connected to SPEED SET pin. In the design, this pin is controlled by the DAC, which is integrated in C8051F330 microcontroller. In this way, the microcontroller is able to easily control the speed fast or slowly.

The speed command is compared with the sensed speed from SPEED FB pin through a transconductance error amplifier. The output of the speed error amplifier is SPEED COMP pin. SPEED COMP is clamped between one diode drop above 3.9V (approximately 4.6V) and one diode drop below $1.7 \mathrm{~V}$ (approximately $1 \mathrm{~V}$ ) to prevent speed loop "wind-up". Speed loop compensation components are connected to this pin.

The voltage on SPEED COMP pin is compared with a ramp oscillator to create a PWM duty cycle [9]. The PWM ramp oscillator creates a sawtooth function from $1.7 \mathrm{~V}$ to 3.9V. A negative clamp at one diode drop below $1.7 \mathrm{~V}$ (approximately 1V) starts the oscillator on power up. The frequency of the ramp oscillator is set by a capacitor to ground $\mathrm{C}_{\mathrm{T}}$ and is selected using the following equation:

$$
C_{T}=\frac{\frac{1}{f_{P W M}} \times 50 \mu \mathrm{A}}{2.4 \mathrm{~V}}
$$

In Equation (3), $f_{\mathrm{PWM}}$ is the PWM frequency in Hz. The PWM duty cycle from the speed control loop is gated the current limit one shot that controls the LA, LB, and LC output drivers.

3) Braking

When the nBRAKE pin is pulled below $1.4 \mathrm{~V}$, the low side output drivers LA, LB, and LC are turned on and the high side output drivers HA, HB, HC are turned off. Braking causes rapid deceleration of the motor and current limiting is deactivated. nBRAKE pin has an internal $4 \mathrm{k} \Omega$ pull-up and can be driven by a switch to ground, an open collector or drain logic signal, or a TTL logic signal.

In the design, nBRAKE is connected with a pin of C8051F330 microcontroller to be driven.

\section{B. Protection of Motors}

\section{1) Current Limiting}

The current sense resistor $\left(\mathrm{R}_{\mathrm{SENSE}}\right)$ Fig. 4 regulates the maximum current in the power stage and the BLDC motor. Current regulation is accomplished by shutting off the output drivers LA, LB, and LC for a fixed amount of time if the voltage across RSENSE exceeds the current limit threshold. $\mathrm{I}_{\text {LIMIT }}$ The voltage on the $\mathrm{I}_{\text {LIMIT }}$ pin sets the current limit threshold. The ML4425 has an internal voltage divider from $\mathrm{V}_{\mathrm{REF}}$ that sets a default current limit threshold of $2.3 \mathrm{~V}$. An external voltage divider referenced to $\mathrm{V}_{\mathrm{REF}}$ can be used to override the default $\mathrm{I}_{\text {LIMIT }}$ setting. The external divider should have at least 10 times the current flow of the internal divider.

The function of $\mathrm{R}_{\mathrm{SENSE}}$ is to provide a voltage proportional to the motor current to set the current limit trip point. The default trip voltage across $R_{\text {SENSE }}$ is $460 \mathrm{mV}$, set by the internal $\mathrm{I}_{\text {LIMIT }}$ divider ratio. In order to pick a value for 
RSENSE, it is necessary to know the maximum current delivered to the motor. This is a function of how much starting torque is required, the acceptable temperature rise in the windings, the rotational losses and the maximum permissible magnetic field. If the motor is sized correctly, the maximum current will be the current necessary to start the motor against its maximum load. The current sense resistor should be a low inductance resistor such as a carbon composition. For resistors in the milliohms range, wirewound resistors tend to have low values of inductance. $\mathrm{R}_{\mathrm{SENSE}}$ should be sized to handle the power dissipation.

This is given by the starting torque divided by the torque constant. Choosing RSENSE to give a maximum current of IMAX yields the following equation:

$$
R_{\text {SENSE }}=\frac{0.46}{I_{M A X}}
$$

$\mathrm{I}_{\mathrm{MAX}}$ is the maximum motor current. The power dissipation is obviously $\mathrm{I}_{\text {MAX }}$ squared times $\mathrm{R}_{\mathrm{SENSE}}$, so the resistor should be sized appropriately.

2) Undervoltage Lockout

Undervoltage lockout is used to protect the 3-phase bridge power stage from a low VDD condition. Undervoltage is triggered at VDD of $9.5 \mathrm{~V}$ or less and is indicated by a TTL low output on the UV FAULT pin. Undervoltage lockout also turns off all output drivers (LA, LB, LC, HA, $\mathrm{HB}$, and $\mathrm{HC})$. The comparator that triggers undervoltage lockout has $150 \mathrm{mV}$ of hysteresis.

\section{Software in Controlling}

As mentioned above, C8051F330 microcontroller is able to receive the commands from users by buttons. Then the microcontroller would output the necessary signals, i.e. speed setting signal and braking signal. Afterwards, the further processing will be done by ML4425. And the parameters are displayed in the LCD to provide users with information of instrument. The flow diagram of software is shown in Fig. 5.

\section{CONCLUSION}

With increasing popularity of BLDC motors used in portable ventilators, they offer significant energy efficiency improvements, lower acoustic noise and better reliability to name a few advantages. According to the principle of motor control, the ML4425 is able to meet the all requirements of BLDC motors. This can provide sensorless commutation plus on chip PWM speed control. An integrating back EMF sensor, combined with an on-chip VCO and sequencer, form a phase locked commutation loop. Therefore, the design has advantages of significant efficiency improvements, lower acoustic noise and better reliability.

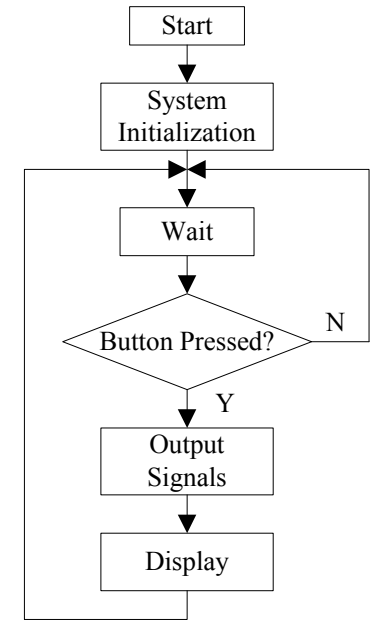

Figure 5. The flow diagram of software.

\section{ACKNOWLEDGMENT}

This work is supported by Zhejiang Bud Talent Plan (2011R405018), Ningbo University Postgraduate Key Course Construction Project (YJ2011KC03), and Ningbo University Experiment Technology Research Project (SYJS201201).

\section{REFERENCES}

[1] Austin PN, Campbell RS, Johannigman JA, and Branson RD, "Work of Breathing Characteristics of Seven Portable Ventilators, " Resuscitation, vol. 49, May. 2001, pp. 159-167.

[2] Emergency Care Research Institute, "Portable/Transport Ventilators," Health Devices, vol. 33, Nov. 2004, pp. 381-401.

[3] Weizi Wang, Fengfeng Xian, Min Wang, and Shunrong Zhou, "A Sensorless BLDCM Speed-adjustment System Based on DSP," Small \& Special Machines, vol. 7, Jul. 2004, pp. 20-22.

[4] Min Chen, Ying Deng, "Brushless DC Motor Control System Based on LPC2141," Microcontrollers \& Embedded Systems, vol. 1, Jan. 2010, pp. 32-34.

[5] Micro Linear, "ML4425/ML4426 Sensorless BLDC PWM Motor Controller," Datasheets of Micro Linear Products, 1997.

[6] Micro Linear, "Application note 43, using the ML4425/ML-4426 BLDC motor controller", Application Notes of Micro Linear Products, 1996.

[7] ATMEL, "AVR444: Sensorless Control of 3-phase Brushless DC Motors," Application Notes of ATMEL Products, 2005.

[8] STMicroelectronics, "AN1946: Sensorless BLDC Motor Control and BEMF Sampling Methods with ST7MC," Application Notes of STMicroelectronics, 2007.

[9] Chunliang Lin, Hornyong Jan, and Niahnchung Shieh, "GA-Based Multiobjective PID Control for a Linear Brushless DC Motor," IEEE/ASME Transactions on Mechatronics, vol. 8, Mar. 2003, pp. $56-65$ 